\title{
An Evaluation of the effectiveness of Barron Band Ligation as a Modality of Treatment of Hemorrhoids
}

\author{
Bharat Chandra ${ }^{1}$, Karthik K S ${ }^{2}$, Seema Kumari ${ }^{3}$, Rajesh Kumar Suman ${ }^{4}$ \\ 1. Surgeon, Department of Surgery, Mysore Medical College and Research Institute, Mysore \\ 2. Surgeon, Department of Surgery, Mysore Medical College and Research Institute, Mysore \\ 3. Medical Officer, Sri Krishna Medical College and Hospital, Muzaffarpur \\ 4. tutor, Department of Pharmacology, MGM Medical College \& Hospital, Navi Mumbai
}

\begin{abstract}
:
Objective: The objective of the study is to evaluate the effectiveness of rubber band ligation in the treatment of internal hemorrhoids.

Methods: This is a prospective study of 120 cases who underwent rubber band ligation as an outpatient procedure for internal haemorrhoids. Binomial probability pre-post test were used for statistical analysis, pvalue less than 0.0001 was taken as significant.

Results: One hundred twenty patients underwent rubber band ligation as a outpatient procedure for internal haemorrhoids. No major post procedure complications as sepsis, vasovagal reflex were observed. Only 36 patients had moderate discomfort following procedure, which lasted for 3-4 days and none had severe discomfort, and 84 patients did not need pain relief; 84 patients returned to work just after procedure of symptom improvement post procedure compared to at presentation only $49 \%$ patients had bleeding post procedure out of 108 patients who had bleeding at presentation p-value of which was significant as $p$ value $=0.00055,6$ patients had pain post procedure out of 36 patients who had pain at presentation $p$-value $=$ 0.00024 was found significant. None patient after procedure had prolapse out of 24 patients who had prolapse on presentation ( $p$-value $=0.0025)$. No patient had irritation or discharge post procedure ( $p$-value $=0.00036)$. Ninety-six patients assessed rubber band ligation as excellent and 24 patients as of moderate help.

Conclusion: Rubber band ligation for internal haemorrhoids is an effective modality of treatment mainly in $2^{\circ}$ grade haemorrhoid which is convenient, simple, high patient acceptance with limitations as post procedure discomfort and pain.
\end{abstract}

Key Words: Rubber band ligation; Sepsis; Vasovagal reflex; Bleeding, Prolapse; Irritation; Discharge

\section{Introduction}

Hemorrhoids are one of the most common benign anorectal problems worldwide. However it has been met with a mixture of sceptism and interest. Haemorrhoids are one of the most common ailments to afflict mankind, For centuries the human race has been plagued by haemorrhoides, but it is impossible to give an accurate figure for their prevalence. Haemorrhoids have plagued humans since they attained the erect posture. The word 'Haemorrhoid' is derived from Greek word Haemorrhoids, meaning flowing of blood (haem=blood, rhoos=flowing). The word 'piles' comes from Latin word pila meaning a pill or ball. To be accurate, we should call the disease as piles when the patient complains of a swelling and 'haemorrhoids' when he or she complains of bleeding'. The treatment of Haemorrhoids has been of interest to surgeons since the Babylonian era. Barron Band Ligation of Haemorrhoids was introduced by Barron (1963) who recommended a modification of the outpatient technique first described by Blaisdell (1958) this procedure reduce the tissue responsible for the production of symptom by strangulation of the redundant mucosa. If Rubber Band Ligation is well tolerated by patients with low incidence of complications and an acceptable recurrence rate, it would be an ideal technique a treatment. There are financial advantage to both patient and country by treating symptomatic Haemorrhoids ${ }^{2}$.

\section{Objectives}

1. To know the effectiveness of Barron Band Ligation in the treatment of hemorrhoids

2. To know the complications associated with Barron Band Ligation.

3. To know the limitations of Barron Band Ligation. 


\section{Material And Methods}

1. Place of study: The present study was conducted in the Department of S u rge ry in K.R. Hospital, Mysore, attached to Mysore Medical College and Research Institute, Mysore, over the duration from January 2011 to July 2012.

2. Study Type: The study was prospective study of evaluation of effectiveness of rubber band ligation in internal hemorrhoids. The study was approved by Institutional Ethical Committee.

3. Sample Size: 120 cases of hemorrhoids were chosen with complaints of bleeding per rectum, pain during detection, mass per rectum, discharge and irritation.

4. Willing patients were selected and examined and investigated as per proforma. The detailed history of each patient was taken with personal history, family history, diet history. Analysis was made on the basis of percentage, mean, standard deviation and binomial probability tests.

5. Procedure: Rubber band ligation was done in minor operation theatre for this study.

6. Preparation: Soap water enema was given to evacuate the rectum on the night before and the morning of the planned day of procedure.

7. Position: Patient was put in left lateral (SIMS) position while the procedure was performed.

8. Principle: Rubber bands applied to the pedicle of mucosa of internal haemorrhoids causes ischaemia, necrosis and scarring preventing further bleeding or prolapse of the respective haemorrhoids and gradually cuts off through the tissues and within a period of seven to ten days these haemorrhoids slough off.

\section{Rubber bands (Barron Bands)}

Black rubber bands with inside diameter of $1 / 16$ th an inch are slide into the inner cylinder of the ligating drum were used. cushion is made to prolapse into the lumen so that the base of the cushion is easy to recognize. The haemorrhoids can be grasped with the alligator forceps and pulled into the cylinder of the ligating drum without causing discomfort. Then with gentle downward traction with alligator forceps and upward pressure with ligating drum the inner cylinder fills with haemorrhoid and underlying vascular tissue forming the pedicle. Now the trigger is pressed so that the rubber bands on the inner cylinder of ligating drum strangulate about $1 \mathrm{~cm}$ diameter of tissue.

Age distribution with sex

\section{Results}

The age and sex distribution of the cases who underwent rubber band ligation is follows.

Table 1: Age and sex distribution

\begin{tabular}{|c|c|c|c|c|c|c|}
\hline \multirow{2}{*}{$\begin{array}{l}\text { Age } \\
\text { group } \\
\text { (years) }\end{array}$} & \multicolumn{2}{|c|}{ Male } & \multicolumn{2}{c|}{ Female } & \multicolumn{2}{c|}{ Total } \\
\cline { 2 - 7 } & $\begin{array}{l}\text { Number of } \\
\text { cases }\end{array}$ & $\begin{array}{l}\text { Number of } \\
\text { cases }\end{array}$ & $\begin{array}{l}\text { Number of } \\
\text { cases }\end{array}$ & \% \\
\hline$\leq 25$ & 9 & 10 & - & - & 9 & 7.5 \\
\hline $26-30$ & 18 & 20 & 12 & 40 & 30 & 25 \\
\hline $31-35$ & 15 & 16.7 & 6 & 20 & 21 & 17.5 \\
\hline $36-40$ & 12 & 13.3 & 8 & 26.2 & 20 & 16.7 \\
\hline $41-45$ & 27 & 30 & - & - & 27 & 22.5 \\
\hline $46-50$ & 6 & 6.7 & 4 & 13.8 & 10 & 8.3 \\
\hline $51-55$ & 3 & 3.3 & - & - & 3 & 2.5 \\
\hline Total & 90 & 100 & 30 & 100 & 120 & 100 \\
\hline Mean \pm SD & \multicolumn{2}{|c|}{$36.33 \pm 8.14$} & \multicolumn{2}{|c|}{$34.33 \pm 6.81$} & \multicolumn{2}{|c|}{$35.83 \pm 7.85$} \\
\hline
\end{tabular}

In the present study the minimum age recorded is 20 years and the maximum age recorded is 60 years. The male female ratio of patients is $3: 1$ respectively. In the present study the mean age for males was 36.33 years with a standard deviation of 8.14 , whereas in females the mean age was 34.33 years with a standard deviation of 6.81 . The overall mean age was 35.83 years with a standard deviation of 7.85 . In the present study, $90(75 \%)$ were males and $30(25 \%)$ were females. 


\section{Heredity}

In the present series the incidence of haemorrhoids in parents (either father or other) is as follows.

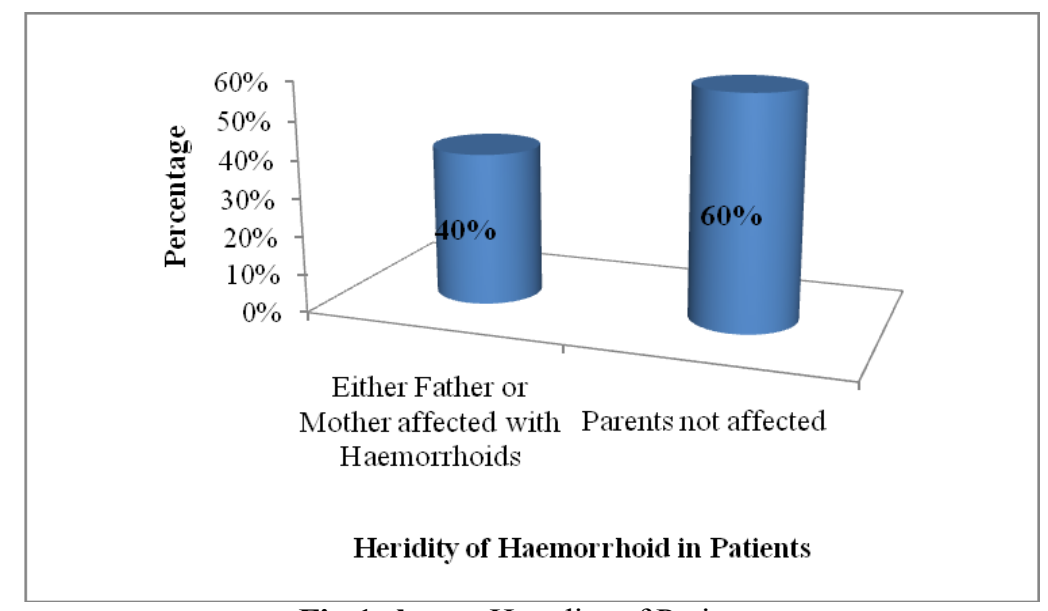

Fig 1 shows: Heredity of Patients

Either father or mother affected with hemorrhoids Parents not affected

In the present study $40 \%$ of patients had significant family history of either father or mother affected by haemorrhoids and $60 \%$ of patients had no any significant parent history.

\section{Bowel habits and straining at stool}

The bowel habits of the present study were grouped under normal or with history of constipation or straining at stool.

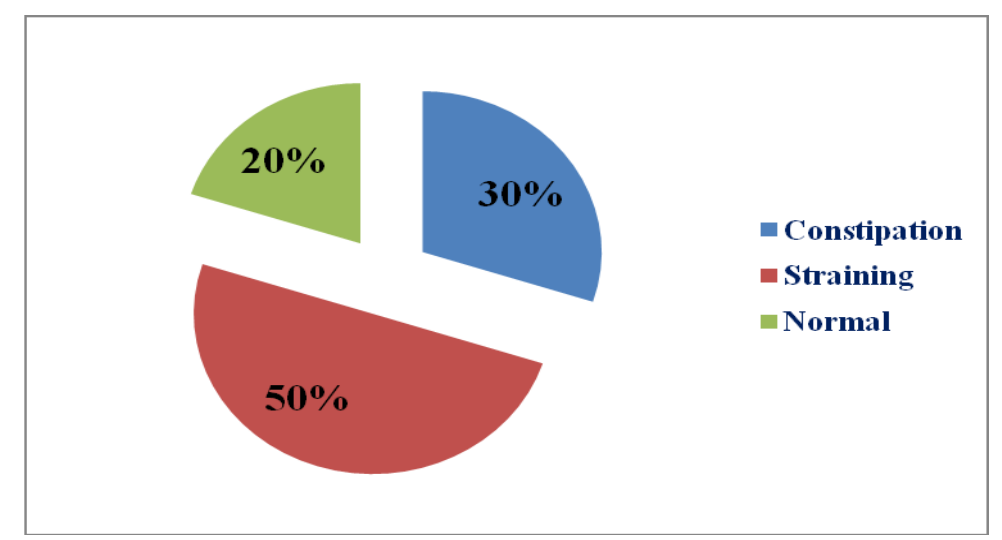

Fig2 shows: Bowel habits and Straining at stool

It was observed that $30 \%$ of cases were constipated whereas $50 \%$ cases had history of straining at stools. Normal bowel habits were seen in only $20 \%$ cases. Maximum percentage i.e., $50 \%$ had history of straining at stools and 30\% had history of constipation and $20 \%$ had history of normal bowel habits. 
Diet

Most of the patients i.e., about $75 \%$ of the subjects were on mixed diet consuming low fibre mostly non-vegetarian diet and $25 \%$ were on vegetarian diet containing high fiber diet.

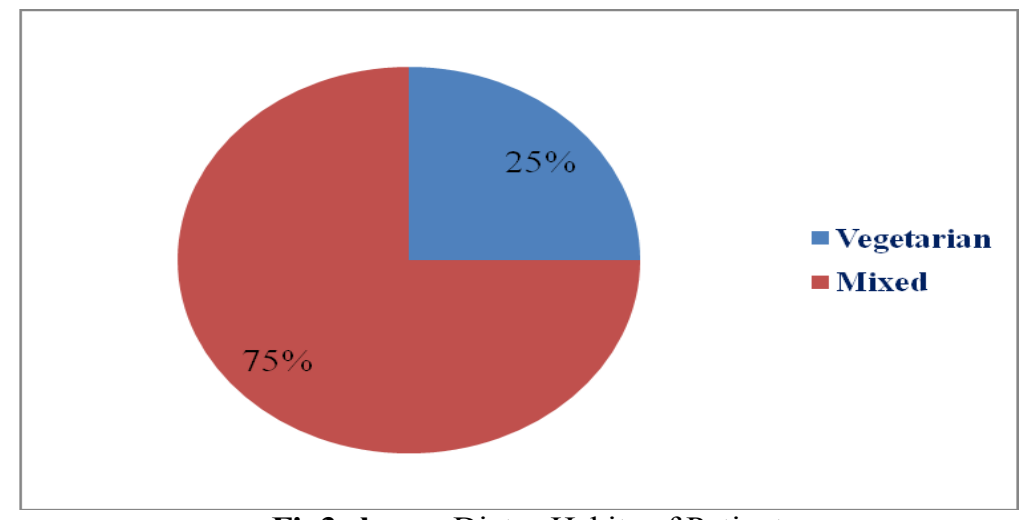

Fig3 shows: Dietry Habits of Patients

\section{Hemoglobin}

The cases were allotted to groups as $<10 \mathrm{gm} \%$, signifying anemia and $>10 \mathrm{gm} \%$ as normal. The observation was as follows only $30 \%$ of the observed cases who underwent rubber band ligation were seemed to be anemic.

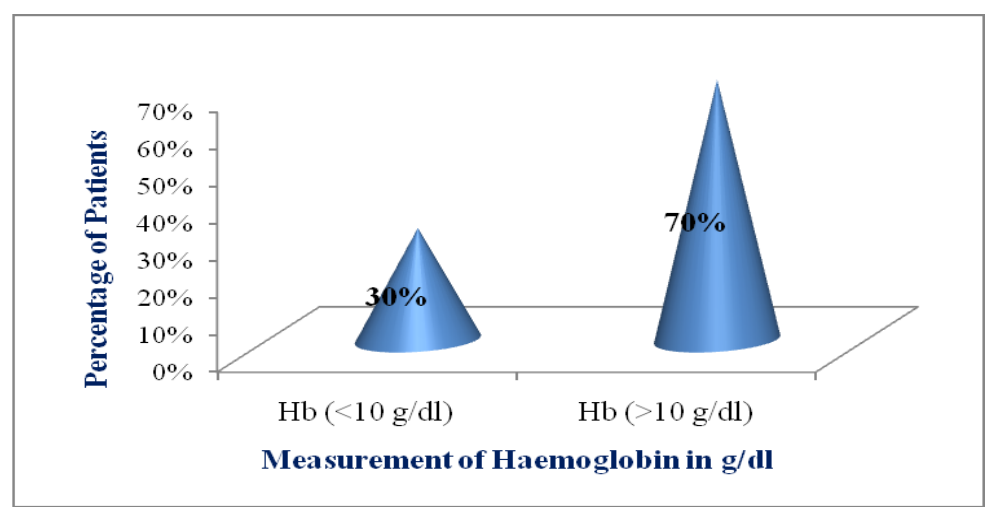

Fig 4 shows: Measurement of Haemoglobin in Haemorrohoids patients

\section{Occupation}

Based on whether the occupation of patients needed them to do manual labourer cases were allotted in manual labourer group or sedentary worker group.About $60 \%$ of patients who underwent rubber band ligation were manual labourers whereas $40 \%$ were sedentary workers. 


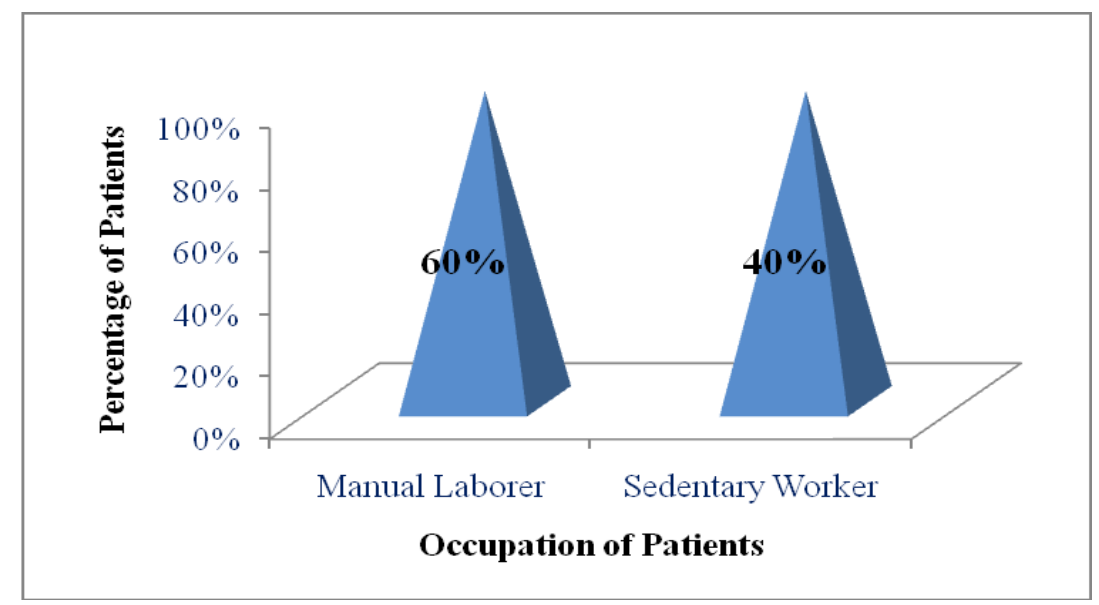

Fig5 shows: Occupation of patients among Hemorrhoids patients

\section{Presenting symptoms}

The presenting symptoms of the 120 cases who underwent rubber band ligation is as follows: Majority of cases i.e., $49 \%$ had bleeding as the predominant symptom, followed by discharge in $9 \%$, pain in $16.21 \%$ cases, irritation in $13.50 \%$ cases and prolapse in $10.82 \%$.

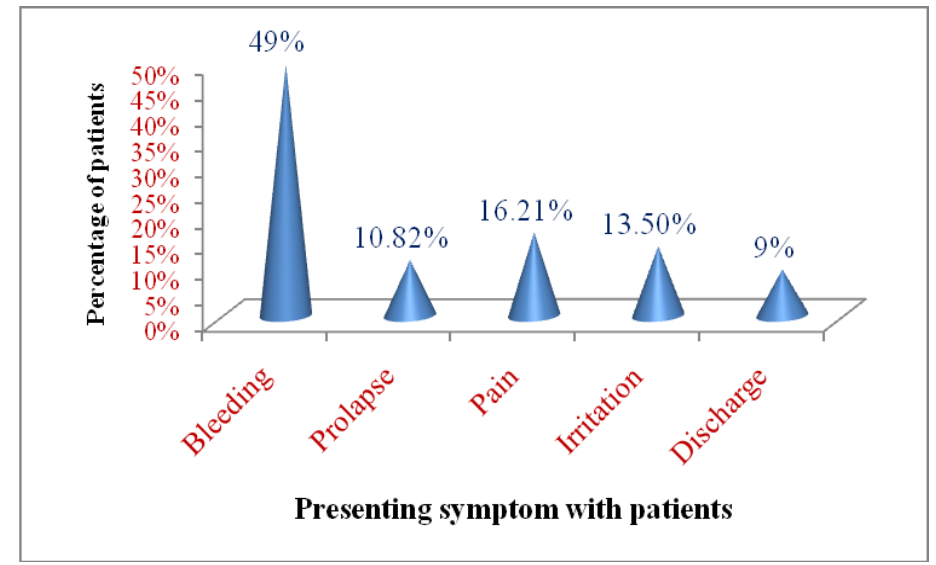

Fig6 shows: Presenting Symptom with Hemorrhoids patients

\section{Immediate post procedure complications}

Following rubber band litigation of the 120 cases, the following post procedure complications were observed in the respective number. Maximum number of cases about $55 \%$ had discomfort, urinary retention was seen in least number of cases around $5 \%$, pain in about $30 \%$ and bleeding about $10 \%$ cases. Complications such as sepsis, vasovagal reflex not seen in the study.

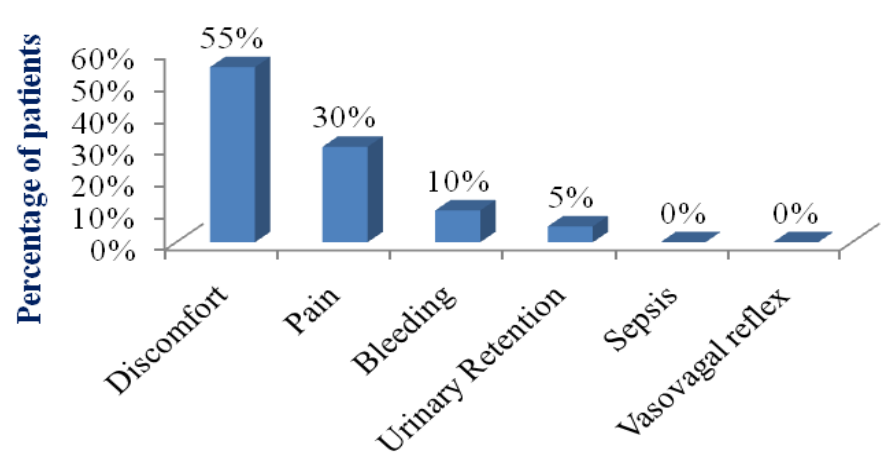

Immediate post Procedure Complication

Fig 7 shows: Immediate post procedure complication in Hemorrhoids patients 


\section{Post ligation discomfort}

Depending on the number of days discomfort was experienced the cases were categorized as slight discomfort lasting about one to two days. Moderate discomfort which was experienced for more than or three days and severe discomfort. None of the cases had severe discomfort whereas maximum patients about $60 \%$ had slight discomfort.

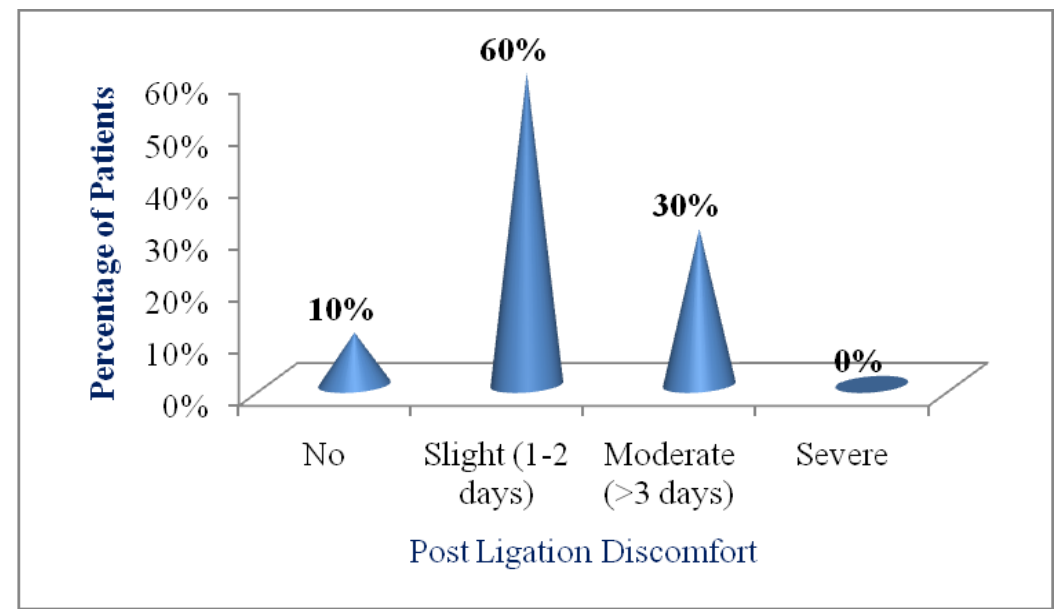

Fig 8 shows: Post ligation Discomfort among Hemorrhoids Patients

\section{Discussion}

Rubber band ligation proved to be a simple technique to acquire and $\mathrm{do}$ not need expertise or too much ski11. Rather no anesthesia, nor pre-procedure elaborate work up. No expensive equipment and no need for bed rest. Patients were made ambulatory and treated as day cases.

\section{Age distribution}

Thoughthenumberof cases was higher in other groups as Khubchandani ${ }^{3}$ with 100 cases and Lee et al. ${ }^{4}$ with 177 cases, the lower age limit of all study groups were around 20 to 25 years whereas the upper age limit was higher in all studies ranging form 70-85 years.

\section{Sex distribution}

In the present study the sex distribution of cases of haemorrhoids were compared 90:30 and Ruffinhood $^{5}$ who showed a 75:25 male:female ratio respectively. Lee performed rubber band ligation for higher number of female cases with a ratio of 52:48, i.e. male:female respectively.

\section{Etiology}

As the etiology is not exactly defined some etiological factors were taken into consideration as occupation, diet, family history, history of constipation, straining at stool. Taking the type of occupation into consideration cases were divided as manual labourers and sedentary workers. There was haemorrhoids preponderance in manual labourers with $60 \%$ cases. This observation is supported by William LJ and Turell who noted that occupational strain and stress played important role in precipitating prolapse of existing internal haemorrhoids ${ }^{6}$.

In the present study $40 \%$ cases had family history of haemorrhoids, which is supported by Greham Stewart's theory of familial tendency due to generalized weakness of venous walls due to hereditary predisposition. Taking into consideration diet, bowel habits and straining at stool. Seventy-five percent of cases were on mixed diet, low on fibre, which was mostly non-vegetarian. Similar to studies, which showed, the close relationship of ids with Western type of diet which is more refined and low in fibre ${ }^{7}$.

This low fibre diet inturn increases bowel transit time and forms hard stools, which causes constipation and straining of stools. Constipation was seen in $30 \%$ cases and straining was seen in $50 \%$ cases. Nivatongs has noted constipation and raised abdominal pressure due to straining as predisposing and associated factors of haemorrhoids ${ }^{7}$. 


\section{Sign \& Symptoms}

The presenting symptoms of the 120 cases who underwent rubber band ligation is as follows in comparison with other studies. The principal presenting symptom in most studies was bleeding per rectum seen in $97 \%$ of Murie et al, ${ }^{8} 88 \%$ of Arabi et al, ${ }^{9} 62 \%$ of David Marshman's study ${ }^{10}$ and $91 \%$ of David Steinberg, ${ }^{11}$ prolapse was seen in $10 \%$ of the present study. $36 \%$ of Murie, $55 \%$ of Arabi, $20 \%$ of David Marshman ${ }^{10}$ study and $64 \%$ of David Steinberg. ${ }^{11}$ Pain in the present study was $30 \%$ comparable with $15 \%$ in David Marshmans. Murie et al. ${ }^{8}$ and Steinberg ${ }^{11}$ had $46 \%$ and $43 \%$ respectively. Arabi et al ${ }^{9}$ had a maximum about $62 \%$ pain. Irritation in the present study was $25 \%$ comparable to Arabi et al. ${ }^{9}$ and Steinberg ${ }^{11}$ whereas Murie et al ${ }^{8}$ had very high of $56 \%$ and Marshman had a very low of $3 \%$. The percentage of patients with discharges was $20 \%$ comparable to Arabi et al ${ }^{9}$ with $29 \%$ and Steinberg with $23 \%$ whereas Murie et al. ${ }^{8}$ had a maximum of $58 \%$.

\section{Treatment}

All 120 cases underwent rubber band ligation as a outpatient procedure with no anaesthesia. Multiple Haemorrhoids were banded in a single session as it was earlier tried by Lee et al, ${ }^{4}$ and Khubchandani. ${ }^{3}$

\section{Immediate post procedure complication}

The cases were watched for immediate complications and compared with studies of Paulvannan and Kumar $^{12}$ and Lee et al. ${ }^{4}$

Maximum patients about $50 \%$ in present study were comparable with Paulvannan and Kumar ${ }^{12}$ study had just $21 \%$ discomfort, pain was noted in $30 \%$ in the present study and $29 \%$ in Paulvannan and Kumar. ${ }^{12}$ Ten per cent of the cases of present study had bleeding comparable to $11 \%$ of Lee ${ }^{4}$ whereas only $1 \%$ Paulvannan and Kumar $^{12}$ had bleeding. $5 \%$ of cases in the present study had urinary retention comparable to $12 \%$ in Lee ${ }^{34}$ study and labours $8 \%$ in Paulvannan and $\mathrm{Kumar}^{12}$. No case of sepsis or vaso-vagal reflex was seen in the present study.

\section{Post-ligation discomfort}

Post rubber band ligation vague discomfort was experienced by most patients, which lasted for about three days. This discomfort categorized as no discomfort, slight discomfort lasting about one to two days. Moderate discomfort, which lasted upto 3 days, was compared with other studies. Maximum patients $60 \%$ in present study and $62 \%$ in both Murie et al. ${ }^{8}$ and Anthony R Groves ${ }^{13}$ study had slight discomfort lasing for 1-2 days were comparable. Moderate discomfort in present study was seen in $20 \%$ cases comparable to $33 \%$ in Murie et al. ${ }^{8}$ and $40 \%$ in Poons. ${ }^{14}$ No discomfort was seen in a minimum of $10 \%$ in present study comparable to $4 \%$ in Murie et al. ${ }^{8}$ and $12 \%$ of Groves study. None had severe discomfort in the present study.

\section{Long-term results}

Though the present study followed up patients only for six months. There have been studies evaluating the long term results of rubber band ligation as David M Steinberg, ${ }^{11}$ who followed up the cases for 4.8 years and reported $89 \%$ as cured or satisfied with results of treatment and Murie et $\mathrm{al}^{8}$ who followed up for 42 months post procedure and found favourable results. 


\section{Summary \& Conclusion}

A hemorrhoid is one of the oldest diseases suffered by mankind, though most of the times not life threatening still it causes significant discomfort.

Though different modalities of treatment are recommended for internal hemorrhoids like rubber band ligation, cryotherapy, bi-polar and direct current therapy. Sclerotherapy, infrared photocoagulation and surgical procedures like Lords anal dilatation, lateral sphincterotomy, haemorrhoidectomy. The search for a simple, outpatient based, convenient cost effective treatment still continues.

The present study was done to know the effectiveness of rubber band ligation in the treatment of internal hemorrhoids. In the present study bleeding was the principle presenting symptoms seen in $90 \%$ cases, followed by prolapse seen in $20 \%$ and irritation in $25 \%$ cases. In the etiology straining at stools was seen in $50 \%, 75 \%$ cases were on mixed diet and $60 \%$ were manual labourers.

There was no serious complications post procedure, vague discomfort was noted in $50 \%$ case with bleeding in only $10 \%$ cases. The study revealed slight post ligation discomfort in $60 \%$ cases, which lasted for $1-2$ days.

\section{Bibliography}

[1]. Keighley and Williams. Surgery of anus, rectum and colon. $3^{\text {rd }}$ ed. Vol 1. Philadelphia: Saunders Publications; 2008. p.351-422.

[2]. Jeffery PJ, Sheila M Ritchie, Miller W, Haweley PR. The treatment of haemorrhoids by rubber band ligation at St Mark's Hospital. Postgraduate Medical Journal 2002;56:847-9.

[3]. Indru $\mathrm{T}$ Khubchandani. A randomized comparison of single and multiple rubber band ligations. Dis Colon Rectum $1983 ; 26: 705-8$.

[4]. Henry H Lee, Robert J Spencer, Robert W Beart Jr. Multiple Haemorrhoidal bandings in a single session. Dis Colon Rectum $1994 ; 37 ; 37-41$.

[5]. Ruffinhood T, Alexander Williams J. Anal dilatation versus rubber band ligation for internal Haemorrhoids. Method of treatment in outpatients. Am J Surg 1971 Oct;122 545-8.

[6]. Warshaw LJ, Turell R. Occupational aspects of proctological disease. New York State Journal of Medicine 1957;57:3006.

[7]. Thomson WH. Haemorroids. Chapter 20.1. In: Oxford textbook of surgery, Morris PJ, Malt RA, eds. New York: Oxford University Press; 1994. p.1125-36.

[8]. MurieJA, SimAJW, MackenzieI. Rubber band ligation versus haemorrhoidectomy for prolapsing Haemorrhoids. A long term prospective clinical trial. Br J Surg 1982;69:536-8.

[9]. Arabi Y, Gatehouse D, Alexander J Williams, Keighley MRB. Rubber band ligationorlateralsubcutaneoussphincterotomy for treatment of haemorrhoids. Br J Surg 1977;64:737-40.

[10]. David Marshman, Philip J Huber Jr, Timmerman W, Thomas C Simonton, Clark F Odom, Eric R Kaplan. Haemorrhoidal Ligation. A review of efficacy. Dis Colon Rectum 1989;32:369-77.

[11]. David M Steinberg, Liegois H, Alexander J Williams. Long-term review of results of rubber band ligation of Haemorrhoids. Br J Surg 1975;62:144-6.

[12]. Kumar N, Paulvannan S, Billings PJ. Rubber band ligation of Haemorrhoids in the outpatient Clinic. Annals of Royal College of Surgery 2002;84:172-4.

[13]. Anthony R Groves, John CW Evans, Alexander J Williams. Management of Internal Ha e morrhoids by Rubber Band Ligation. Br J Surg 1971 Dec;58(12):923-4.

[14]. Poon GP. Conventional Vs. Triple Rubber band ligation of Haemorrhoids. A prospective randomized trial. Dis Colon Rectum $1986 ; 836-8$ 